Review

International Journal of Biological Sciences

ISSN 1449-2288 www.biolsci.org 2008 4(5):318-329

(C) Ivyspring International Publisher. All rights reserved

\title{
Unveiling the Bmp13 Enigma: Redundant Morphogen or Crucial Regulator?
}

\author{
Lisa A Williams ${ }^{凶}$, Divya Bhargav, Ashish D Diwan \\ Spine Service, St George Clinical School, University of New South Wales, Sydney, Australia \\ $\checkmark$ Correspondence to: Dr Lisa A Williams, 1.williams@spine-service.org; Tel: +61 29567 5888; Fax: +61 295679411
}

Received: 2008.07.30; Accepted: 2008.09.08; Published: 2008.09.11

\begin{abstract}
Bone morphogenetic proteins are a diverse group of morphogens with influences not only on bone tissue, as the nomenclature suggests, but on multiple tissues in the body and often at crucial and influential periods in development.

The purpose of this review is to identify and discuss current knowledge of one vertebrate BMP, Bone Morphogenetic Protein 13 (BMP13), from a variety of research fields, in order to clarify BMP13's functional contribution to developing and maintaining healthy tissues, and to identify potential future research directions for this intriguing morphogen. BMP13 is highly evolutionarily conserved (active domain $>95 \%$ ) across diverse species from Zebrafish to humans, suggesting a crucial function. In addition, mutations in BMP13 have recently been associated with Klippel-Feil Syndrome, causative of numerous skeletal and developmental defects including spinal disc fusion. The specific nature of BMP13's crucial function is, however, not yet known.

The literature for BMP13 is focused largely on its activity in the healing of tendon-like tissues, or in comparisons with other BMP family molecules for whom a clear function in embryo development or osteogenic differentiation has been identified. There is a paucity of detailed information regarding BMP13 protein activity, structure or protein processing. Whilst some activity in the stimulation of osteogenic or cartilaginous gene expression has been reported, and BMP13 expression is found in post natal cartilage and tendon tissues, there appears to be a redundancy of function in the BMP family, with several members capable of stimulating similar tissue responses. This review aims to summarise the known or potential role(s) for BMP13 in a variety of biological systems.
\end{abstract}

Key words: BMP13, Cartilage; Growth Factor; Development; Bone

\section{Introduction}

Bone Morphogenetic Protein 13 is a member of the wider Bone Morphogenetic Protein (BMP) family, a group of bio-active growth factors with $20-30 \%$ amino acid homology to Transforming Growth Factor $\beta$ (TGF $\beta$ ). Initially BMPs were identified as components of de-mineralised bone matrices which stimulate the generation of new bone tissue when implanted ectopically, or at sites of fracture [1,2]. BMP13 was identified in cartilaginous tissues, and by virtue of its homology to other members of the BMP family [3,4].

More than 30 BMPs have now been identified in a wide range of species. Protein structure and, to a lesser degree amino acid sequences are evolutionarily conserved; and BMPs are present in diverse species such as mammals, fish, amphibians and birds. BMP-like molecular pathways have been identified in invertebrates such as Drosophila [5-7] and in the nematode Caenorhabditis elegans [8,9]. To date BMP13 appears to be vertebrate specific [10].
Members of the BMP family have a range of $40-60 \%$ amino acid identity but can be divided into subgroups on the basis of structural and amino acid similarities, as listed in Table 1. BMP13 (GDF6, CDMP2) has a higher degree of homology (80-86\% amino acid identity) with other members of the GDF sub family (GDF5/BMP14, GDF7/BMP12) than with the wider BMP group (about 50\%) [11-13], indicating the potential for conserved function.

Table 1. BMP protein family sub-grouping by amino acid homology (*Homology in C-terminal active domain).

\begin{tabular}{lll}
\hline BMP sub-group & $\begin{array}{l}\text { Amino acid homology to } \\
\text { BMP13 sub-group* }\end{array}$ & Alternative Name \\
\hline BMP-2 & $56-57 \%$ & $\begin{array}{l}\text { BMP2A } \\
\text { BMP-4 }\end{array}$ \\
BMP-6 & $50-54 \%$ & BMP2B \\
\hline BMP-7 & & Ogr-1 \\
BMP-5 & & OP-1 \\
BMP-8 & & OP-2 \\
BMP-3 & $46-47 \%$ & Osteogenin \\
\hline BMP-12 & $82 \%$ & GDF-7 / CDMP-3 \\
BMP-13 & $100 \%$ & GDF-6 / CDMP-2 \\
\hline BMP-14 & $82 \%$ & GDF-5 / CDMP-1 \\
\hline
\end{tabular}


BMPs are considered to be pleiotropic, effecting many different tissues in subtly different ways. They have been identified and characterized through diverse fields of biology such as tissue healing, regeneration and maintenance $[3,11,12,14]$; mouse developmental mutational analysis $[4,15,16]$; Zebrafish and Xenopus embryo development [17-19]; and investigations of human developmental anomalies [20,21]. BMPs play crucial roles in early embryonic patterning and in skeletal and organ development [22-25].

\section{BMP13 Protein Structure}

The amino acid sequence for BMP13/GDF6 is highly conserved across vertebrates, although to date no invertebrate orthologues have been identified [10]. Sequence homology is concentrated in the active C-terminal domain (Figure 1) indicating crucial conservation of function. Even the most divergent of BMP13-homologues, Zebrafish dynamo and radar, display $>90 \%$ homology in this domain. Considerably less sequence similarity is found in the N-terminal pro-domain, although there are conserved regions in higher vertebrates associated with developmental mutations [21] (Figure 2).

$\mathrm{H}=$ Human, $\mathrm{M}=$ mouse, $\mathrm{B}=$ Bovine, $\mathrm{X}=$ xenopus, $\mathrm{D}=$ Dynamo (Zebrafish), $\mathrm{R}=$ radar (Zebrafish), $\mathrm{r}=$ Rhesus Macaque

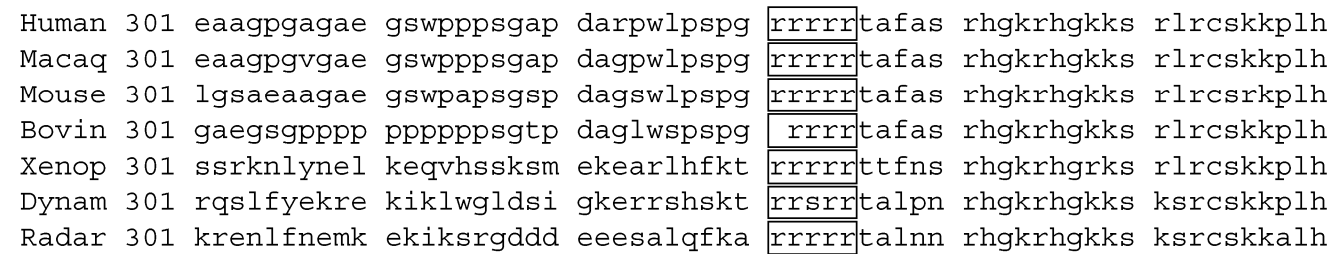

Human 361 vnfkelgwdd wiiapleyea yhcegvcdfp lrshleptnh aiiqtlmnsm dpgstppscc Macaq 361 vnfkelgwdd wiiapleyea yhcegvcdfp lrshleptnh aiiqtlmnsm dpgstppsce Mouse 361 vnfkelgwdd wiiapleyea yhcegvcdfp lrshleptnh aiiqtlmnsm dpgstppscc Bovin 361 vnfkelgwdd wiiapleyea yhcegvcdfp lrshleptnh aiiqtlmnsm dpgstppscc Xenop 361 vnfkelgwdd wiiapleyea hhcegvcdfp lrshleptnh aiiqtlmnsm npgstppscc Dynam 361 vnfrelgwdd wviapldyea yhcegmcdfp lrshleptnh aiiqtlmnsm npsnmppscC Radar 361 vnfkelgwdd wiiapldyea yhcegvcdfp lrshleptnh aiiqtlmnsm dpnstppscc

Figure 1. Alignment of the BMP13 C-terminal active domain amino acid sequence from various vertebrate species. Shaded areas represent amino acid differences compared to the human sequence. Consensus protease cleavage site (boxed). Accession numbers for sequences: Human-NM_001001557; Rhesus Macaque - XM_001090825; Mouse-MGI95689; Rat-NM_001013038; Bovine-U13661; Xenopus-AAD38402; Radar (Zebrafish)-AAB34226; Dynamo (Zebrafish)-X99769.

\begin{tabular}{|c|c|c|}
\hline uman & \multicolumn{2}{|c|}{ lrhqpwkqlclelraawg-eldageaearargpqqppppdlrslgfgrrvrppqerallvvftrsq-rknlfaemreql } \\
\hline Macaq & rhqpwkqlclelraaws & rgpqqppppdlrslgfgrrvrppqerallvvftrsq-rknlfaemreq \\
\hline Iouse & lrpqpwkqlclelraaws & rgpqqpppldlrslgfgrrvrppqerallvvftrsq-rknlftemhec \\
\hline attu & lrpqpwkglclelravws & rgpqqsppldlrslgfgrrvrppqerallvvftrsq-rknlftemhec \\
\hline Bovin & lrpqpwkqlclelraaws & qerallvvfsrsq-rktlfaemrec \\
\hline Xenop & disqgkkqicvelkais & nqekallvvftkss-rkn」ynelkec \\
\hline Radar & khrhqenqlclqlkv & tygksdteidlkqlgfh rhsntqqerailvvytrskkrenl fnemkel \\
\hline ynam & qhhshgnrfclelra & lkkailvvftrskkrqslfyekr \\
\hline
\end{tabular}

Figure 2. Alignment of amino acid sequences surrounding the putative BMP13 second cleavage site. Amino acid sequences alignment demonstrates homology upstream and downstream of a second consensus protease cleavage site (boxed). Residues highlighted in Red are particularly conserved. 


\begin{tabular}{|c|c|c|c|c|c|}
\hline 10 & 20 & 30 & 40 & 50 & 60 \\
\hline MDTPRVLLSA & VFLISFLWDL & PGFQQASISS & SSSSAELGST & KGMRSRKEGK & MQRAPRDSDA \\
\hline 70 & 80 & 90 & 100 & 110 & 120 \\
\hline GREGQEPQPR & PQDEPRAQQP & RAQEPPGRGP & RVVPHEYMLS & IYRTYSIAEK & LGINASFFQS \\
\hline 130 & 140 & 150 & 160 & 170 & \\
\hline SKSANTITSF & VDRGLDDLSH & TPLRRQKYLF & DVSMLSDKEE & LVGAELRLFR & QAPSAPWGPP \\
\hline 190 & 200 & 210 & 220 & 230 & $24 c$ \\
\hline AGPLHVQLFP & CLSPLLLDAR & TLDPQGAPPA & GWEVFDVWQG & LRHQPWKQLC & LELRAAWGEL \\
\hline 250 & 260 & 270 & 280 & 290 & 30 \\
\hline DAGEAEARAR & GPQQPPPPDL & RSLGFGRRVR & PPQERALLVV & FTRSQRKN凹F & AEMREQLGS \\
\hline 310 & 320 & 330 & 340 & 350 & 360 \\
\hline EAAGPGAGAE & GSWPPPSGAP & DARPWLPSPG & RRRRTAFAS & RHGKRHGKKS & RLRCSKKPLH \\
\hline 370 & 380 & 390 & 400 & 410 & 42 \\
\hline VNFKELGWDD & WIIAPLEYEA & YHCEGVCDFP & LRSHLEPTNH & AIIQTLMNSM & DPGSTPPSCC \\
\hline 430 & 440 & 450 & & & \\
\hline VPTKLTPISI & LYIDAGNNVV & YKQYEDMVVE & SCGCR & & \\
\hline \multicolumn{6}{|c|}{$\begin{array}{l}\text { MDTP..... = signal peptide } \\
\text { CC, CC, CC - Intra chain Cystine pairs } \\
\text { C - Cystine Residue binding Inter Chain }\end{array}$} \\
\hline \multicolumn{6}{|c|}{ Protease Cleavage Site Releases Active Domain } \\
\hline \multicolumn{6}{|c|}{ Second Protease Consensus cleavage site } \\
\hline \multicolumn{6}{|c|}{$\begin{array}{l}* \text { putative } N \text {-glycosylation site } \\
\mathrm{K} \text { Natural Variant }-\mathrm{K} \rightarrow \mathrm{E}\end{array}$} \\
\hline $\begin{array}{l}\mathrm{L} \rightarrow \mathrm{P} \\
\mathrm{A} \rightarrow \mathrm{E}\end{array}$ & $\begin{array}{l}\text { KFS mutation a } \\
\text { KFS mutation a }\end{array}$ & $\begin{array}{l}\text { a } 289 \text { familial } 100 \\
\text { a } 249 \text {-sporadic } n\end{array}$ & & & \\
\hline
\end{tabular}

Figure 3. BMP13 Protein Sequence. Amino acid sequence of human BMP13 showing signal peptide, disulphide-bonded cysteine pairs, consensus protease. Cleavage sites and point mutations identified in degenerative conditions.

Protein structural information for BMP13 is speculative, relying on the analysis of conserved structural characteristics of members of the BMP family, and based on studies of BMP4 [26-29] and BMP7 [30]. The 3-dimensional structure of the BMPs is referred to as a "cysteine knot" [31]. Synthesised as large precursor molecules containing a pro-domain and an active domain, BMPs form homodimers in the Endoplasmic Reticulum (ER) and are proteolytically cleaved in the Golgi [32] at dibasic consensus RXXR seropeptidase cleavage sites. BMP13 has a consensus sequence for cleavage by a serine pro-protein convertase such as furin [33], positioned to release an active C-terminal domain of 121aa (Figure 3). Western blotting data under reducing and non-reducing conditions confirms BMP13 exists as a homodimer of approximately $16 \mathrm{KDa}$ subunits [34-37], structurally held together by interand intra-chain disulphide bonds between 7 conserved cysteine residues (see Figure 3). Interestingly the BMP13 amino acid sequence also possesses a second putative dibasic consensus cleavage site upstream in the pro-domain similar to that identified for BMP4
[29], indicating that control of the release of active BMP13 may rely on 2 proteolytic cleavage steps within the ER and Golgi (Figure 2). An amino acid sequence alignment of this region shows considerable homology across diverse species, and encompasses 2 loci where developmental mutations associated with Klippel-Feil Syndrome have been identified [21] (Figure 2).

Evidence obtained from purification of bioactive fractions from bone suggest BMPs, in addition to forming homodimers, can form heterodimeric molecules in vivo [38,39]. In vitro studies have implied that BMP2/7 and BMP4/7 heterodimers formed preferentially and had significantly greater activity in osteogenic differentiation assays $[40,41]$ and in mesoderm induction in Xenopus oocytes [42,43] than the two homodimeric molecules combined. BMP13 formed heterodimers with BMP2 when co-expressed in the same cell in vitro and may also interact with BMP4 [17], however this is yet to be demonstrated in vivo. Indeed, any direct evidence of protein processing, structure or physical characteristics specific to BMP13 are as yet unavailable or unpublished. 
Table 2. BMP13/GDF6/CDMP2 sequence identification.

\begin{tabular}{|c|c|c|c|c|}
\hline Species & Name & Reference & Tissue & Accession No. \\
\hline Bovine & CDMP-2 & Chang et al 1994 & Articular cartilage & U13661 \\
\hline Human & BMP13 & $\begin{array}{l}\text { Strausberg et al 2002, } \\
\text { Asai-Coakwell et al } 2007\end{array}$ & $\begin{array}{l}\text {-mixed } \\
\text {-genomic DNA sequencing } \\
\text { project }\end{array}$ & $\begin{array}{l}\text { AAH4322 } \\
\text { NM } 001001557\end{array}$ \\
\hline Rhesus Macaque & Gdf6 & Lowe \& Eddy 1997 & $\begin{array}{l}\text { Genomic DNA sequencing pro- } \\
\text { ject }\end{array}$ & XM_001090825 \\
\hline Mouse & GDF6 & Storm et al 1994 & $12.5 \mathrm{~d}$ embryo & MGI 95689 \\
\hline Rat & GDF6 & Sena et al 2003 & Mandibular molar & RGD: 620104 \\
\hline Zebra fish & radar & Rissi et al 1995 & Early embryo & AAB34226 \\
\hline Zebra fish & dynamo & Bruneau \& Rosa 1997 & Early Embryo & X99769 \\
\hline Xenopus & GDF6 & Chang \& Hemmati-Brivanlou 1999 & Mid-gastrula embryo & AAD38402 \\
\hline
\end{tabular}

\section{Receptor Transduced Signalling}

BMP13, like all members of the BMP family, functions via cell signaling through trans-membrane serine-threonine kinase receptor complexes [24,44,45] (Table 3). BMP signal receptor complexes contain both Type I (BMPRIA, BMPRIB, ALK1, ALK2) and Type II (BMPRII, ActRII, ActRIIB) receptor molecules. The homodimeric BMP ligand first binds to a constitutively active type II receptor, which recruits and phosphorylates the type I receptor, conferring ligand binding, kinase domain activation and initiating intracellular signalling cascades $[24,45,46]$. The type I receptor molecules are necessary for the signalling component of the complex [47]. BMP receptors are inducible in tissues such as bone, where both BMPRIA/IB increase expression at sites of ossification, and display temporal expression in specific tissues during embryogenesis [45].

BMP13 has demonstrated preferential affinity for BMPRIB (Activin receptor-Like Kinase 6 (ALK6)) and BMPRII. In the rat osteoprogenitor cell line ROB-C26 BMP13 formed complexes with BMPRIB and BMPRII which transduced a strong transcription initiation signal, with a weaker signal and barely detectable receptor complex associated with BMPRIA/BMPRII [34]. Interestingly, the BMPRIB/BMPRII complex is crucial for chondrogenesis of chick limb mesenchymal cells [48], suggesting a role for BMP13 (and closely related GDF family members) in chondrogenic develoment.

The downstream events associated with BMP receptor complex activation involve recruitment and activation of Receptor-associated-Smad signalling molecules (R-Smad), which combine with the common Smad (C-smad), Smad-4, to propagate the BMP receptor signals to the nucleus through their ability to bind to specific DNA sequences and promote gene expression [25,49-52]. BMP13 binding to BMPRIB, BMPRIA and BMPRII results in phosphorylation/activation of Smad 1/5/8, and transcriptional activation via BMP response elements (BRE) in vitro [53]. Blocking BMP13 (GDF6) signalling in vivo results in a reduction in phospho-smad in corresponding regions of the developing embryo [19].

Table 3. BMP Receptor groupings and nomenclature.

\begin{tabular}{l|lll}
\hline Receptor & Alternative Name & Type & BMPs Bound \\
\hline ALK3 & BMP-RIA & I & $2,4,7,13,14$ \\
\hline ALK6 & BMP-RIB & I & $2,4,7,13,14$ \\
\hline ALK1 & & I & \\
\hline ALK2 & Activin RI & I & $2,7,14$, activin \\
& & & \\
\hline BMP-RII & & II & $2,4,7,13,14$ \\
\hline Act-RIIA & & II & $7,2,13,14$, activins \\
\hline Act-RIIB & & II & $2,7,14$, activins \\
\hline
\end{tabular}

\section{BMP13 Expression and Function}

BMP13, was first identified and isolated as a component of bovine cartilage, designated Cartilage Derived Morphogenetic Protein -2 (CDMP2) [3], expressed in post natal articular and cricoid cartilage, and to a lesser degree in other tissues such as intestines, skeletal muscle and placenta. Expression has since been detected in a variety of structural tissues and aspects of its function have been emerging. The majority of studies focus on the promotion of connective tissue healing and on comparisons with other BMPs known for their ability to stimulate the growth of bone. However, a body of literature examines BMP13 and its homologues in embryonic development, work that could provide clues to the more specific roles of BMP13 in adult tissues.

\section{BMP13 and Cartilage}

BMP13 expression has been detected in both foetal and post natal cartilaginous tissues from various species and anatomical sites [3,35,36,54,55]. Expression was detected mainly in the upper layers of post natal articular cartilage tissues [35] but was uniform in cultured chondrocytes. Examination of human foetal tissues (6 - 10 wks gestation) has localized BMP13 expression to mature and hypertrophic chondrocytes in the periosteal bony collar of developing long bones [3].

Chondrocytic cells isolated and cultured in vitro respond to recombinant BMP13 stimulation, increasing the biosynthesis of proteoglycan (PG) [34-36,56,57] 
measured by alcian blue staining, ${ }^{35}$ S-incorporation or as a percentage of tissue wet weight [57]. This appears to be, at least in part, due to increased aggrecan mRNA levels [36], however more data is required as other study results do not show aggrecan mRNA upregulation [35].

BMP13 does not appear to increase chondrocyte or cartilage cell proliferation $[34,36]$, although proliferation was reported in a study of mesenchymal progenitor cell differentiation (discussed further below) [58]. The location of expression in cartilaginous tissues would suggest a presence in proliferating cells.

Surprisingly, BMP13 stimulation of cartilaginous tissues did not demonstrate increased levels of collagen expression $[35,36,56]$, yet collagen II upregulation has been reported in mesenchymal progenitor cells stimulated with BMP13 [58,59]. This is perhaps a reflection of the differentiation state of the cells under study, rather than the signals being transduced by the BMP13 ligand.

The measurement of increased catabolic activity in cells stimulated with BMP13, while indicative of activity, was not specific or limited to BMP13 alone. Indeed all CDMPs (BMP13, 12, 14), when compared directly, induced chondrocytic protein synthesis with varying levels of intensity depending on the tissues studied $[60,61]$.

\section{BMP13 in Osteogenesis}

The potential for BMP13 to stimulate osteogenic phenotypes has been examined [34,57-59,62-64].

Rather than osteogenic differentiation, BMP13 appears to induce marker expression in progenitor cells that are characteristic of chondrocytes, such as proteoglycan $[34,37,57,58,64]$ and collagen II $[58,59]$. BMP13 stimulation of a number of different progenitor cell types had no effect on the expression of osteogenic markers osteocalcin $[57,58,62]$, myoD [63] and calcium mineral accumulation [58] that are characteristic of osteogenic differentiation. Further, in a study of human bony outgrowths seen in joint arthritis and known as osteophytes, BMP13 expression was absent from osteoblasts or newly formed osteocytes, rather localized to the proliferating and mature chondrocytes, and to a lesser degree to hypertrophic chondrocytes [54].

Some ambiguity exists with respect to BMP13 stimulation of alkaline phosphatase (ALP) activity, which is reportedly increased, albeit to low levels, in mesenchymal progenitor cell lines $[34,58,59]$ and BM stromal cells [62] in response to recombinant BMP13 stimulation. However other studies in bovine ligament fibroblasts [37], periosteum [57], and one C2C12 murine progenitor cell line study [63] reported no increase in ALP activity. Whilst the ALP studies utilised similar enzyme activity methodology they were done in different cell types, and there was variability in the incubation times (48h to 1-3 weeks), where the longer stimulation times in general resulted in detection of elevated ALP in response to BMP13. It is clear that any enhancement of ALP activity was inferior to that of the more osteogenic BMP7 and BMP2 molecules, and that whilst osteogenesis was not suppressed by BMP13, it was also not significantly enhanced. Interestingly, BMP13 did synergistically enhance the expression of BMP7-induced ALP, myoD and osteocalcin in the C2C12 murine progenitor cell line [63], as did all of the CDMPs (BMP13, BMP14 (GDF5) and BMP12 (GDF7)) studied in $\mathrm{C} 2 \mathrm{C} 12$ cells- indicating a potential modulator role for BMP13.

These studies also demonstrated that BMP13 showed reciprocal stimulation of mRNA in culture with other BMPs. BMP13 auto-regulated itself and was up-regulated by BMP12 (GDF7, CDMP-3); BMP13 also significantly upregulated the expression of BMP4 mRNA (8-10 fold), and to a lesser degree that of BMP5, GDF8 (myostatin; a negative regulator of skeletal muscle development) and GDF9 (involved in control of ovulation) (2-3 fold), particularly, in the case of GDF8 and 9, following longer incubation periods (6and 11-fold increases respectively). BMP13 was found to suppress the expression of BMP6 and BMP-8A by $60-70 \%$ [63]. Taken together, these data point towards a modulator role for BMP13 growth factor signals in the tissue, with an overall enhancement of cartilaginous growth and down regulation of bone-promoting activities.

Table 4. BMP family members and major functional activities. References: $[65,66]$

\begin{tabular}{|c|c|c|}
\hline BMP & Other names & Known Activity \\
\hline BMP2 & BMP2A & Cartilage/bone morphogenesis; osteoblasts differentiation; retinoid mediator \\
\hline BMP3 & - & Osteogenesis, bone formation; brain \\
\hline BMP4 & BMP2B & $\begin{array}{l}\text { Cartilage/bone morphogenesis; formation teeth, limbs, \& bones from mesoderm; } \\
\text { fracture repair }\end{array}$ \\
\hline BMP5 & - & Bone morphogenesis; cartilage development \\
\hline BMP6 & - & Hypertrophy-cartilage/skin; adult joint integrity \\
\hline BMP7 & OP-1 & $\begin{array}{l}\text { Bone morphogenesis, differentiation; eye and kidney development; osteoblasts } \\
\text { differentiation; }\end{array}$ \\
\hline BMP8 & OP-2 & Bone formation; cartilage development \\
\hline BMP8A & & Germ cells of testis - spermatogenesis \\
\hline
\end{tabular}




\begin{tabular}{l|l|l}
\hline BMP8B & - & Chondrogenic differentiation from mesenchymal progenitors \\
\hline BMP9 & GDF7 & Ligament \& tendon development \\
\hline BMP12 & GDF6 & $\begin{array}{l}\text { Cartilage development, hypertrophy; embryonic patterning; eye development; limb } \\
\text { morphogenesis }\end{array}$ \\
\hline BMP13 & GDF5 & $\begin{array}{l}\text { Limb morphogenesis; chondrogenesis; mesenchymal condensation } \\
\text { Begative regulation of skeletal muscle growth }\end{array}$ \\
\hline & GDF8 & Control of ovulation; expressed in oocytes, ovary \\
\hline
\end{tabular}

\section{BMP13 in Tendons \& Ligaments}

BMP13 expression was detected in human tendon tissues $[67,68]$. Expression was specifically detected at sites of active tissue healing and re-modeling - particularly in the small, rounded tenoblasts, capable of proliferating and synthesizing tendon extracellular matrix proteins; and in perivascular mesenchymal cells thought to act as stem cells for connective tissue healing [68]. In vitro BMP13 stimulation induced collagen I expression and, unlike most cellular studies, very low concentrations of recombinant BMP13 (25 $50 \mathrm{ng} / \mathrm{mL}$ ) were found to stimulate tenoblast proliferation [68]. The active concentration range of BMP13 activity reported in this tendon study has not been duplicated in published studies to date. Whether tenoblasts are particularly responsive to BMP13, or the reagents particularly potent is not known, but clearly the activity was greater than in other published studies which utilise in the vicinity of $200-300 \mathrm{ug} / \mathrm{mL}$ $[34-37,56,59,63,64]$. Interestingly it is one of the only studies published where human recombinant proteins were used to stimulate human tissues.

Several studies have reported neo-tendon/ligament formation and the development of highly organized connective tissue rich in collagen I fibres in response to in vivo ectopic implantation of BMP13 at sites of tendon wounding in rats $[61,69,70]$ and intramuscularly $[60,71]$. Progenitor cell proliferation and differentiation into neo-tendon/neo-ligament was reported within the muscle, the formation of collagenous extracellular matrix, and even development of small bone and cartilage foci within the new tissue [71].

Further in vivo studies demonstrated that BMP13 has significant ability to accelerate the healing of damaged tendons and ligaments in vivo, inducing mechanical strengthening of the tendon fibres which were measurably stronger 8 days following a single injection of recombinant BMP13 [61,70]. Intriguingly, researchers found that implantation at physiological sites subject to different mechanical loading stimulated the growth of either bone tissue (unloaded tendons) or cartilaginous tissue (mechanically loaded tendons) [69].

It is noteworthy that whilst these tendon tissue studies reported stimulation of collagen I fibre forma- tion, isolated bovine ligament fibroblasts stimulated with BMP13 in vitro were induced to express a more chondrogenic phenotype, increasing expression of proteoglycan [37]. Taken together with evidence of differential tissue formation in response to mechanical loading, it appears that BMP13 induction of cellular catabolism produces the up-regulation of different sets of genes in different circumstances.

\section{BMP13 in the Intervertebral Disc}

The structure of the intervertebral disc (IVD) is a gelatinous, highly hydrated core - the Nucleus Pulposus (NP), comprised largely of proteoglycan and collagen II, surrounded by a high tensile strength collagen fibre "fence" in the form of the Annulus Fibrosus (AF).

Studies in cells derived from the Nucleus Pulposus (NP) [72-74] and Annulus Fibrosus (AF) [73], in comparison to other catabolically active BMPs, have demonstrated that BMP13 induced disc cell catabolic activity. BMP13 stimulated increased production of proteoglycan and total collagen in the absence of increased cell proliferation in IVD monolayer cultures $[72,73]$ and in 3D alginate co-culture with transduced chondrocyte monolayers [74]. Furthermore the data suggest that AF cell cultures are more responsive to BMP13 than NP cells, producing proportionately more proteoglycan and collagen in relation to controls and in comparison to other BMPs tested [73].

In addition, $\mathrm{Li}$ et al (2003) have utilized a mouse chondrocytic cell line MC615 to study the potential for BMP13 in combination with BMP2 to stimulate the production of extracellular matrix proteins characteristic of the disc tissue. Whilst this cell line is not derived from the disc it demonstrates similar gene expression. The authors found that both proteins could stimulate the production of proteoglycan, mediated by increased aggrecan mRNA expression, but had little effect on cell proliferation. In this model the stimulatory effect of BMP2 was greater than BMP13, occurred at lower concentrations, and no synergistic activity was detected [64].

We have recently obtained early data from a large animal disc degeneration model [75] which suggests that BMP13, when injected directly into the disc, can reverse early degenerative changes induced by mechanical injury (Diwan et al 2008, unpublished obser- 
vations; Approved by the University of Sydney Animal Ethics committee, August 2007). Our study detected a loss of disc integrity using MRI, where an annulus stab injury receiving saline injection was visible as a darker (more degenerated) T2-weighted image compared to those which received BMP13 injection, or uninjured controls (Figure 4). Histological analyses revealed an increase in the number of cells discharging into the disc from the discal side of the end plate in BMP13-injected discs, compared to the stab-controls or exposed un-injured controls (Figure 5). This was accompanied by evidence of neo-vascularization, a known response to chronic injury and characteristic of this model [76], which we observed on the bony side of the end plate region in disc tissues derived from the stab-only controls, yet not in the BMP13-injected disc tissues (Figure 5).

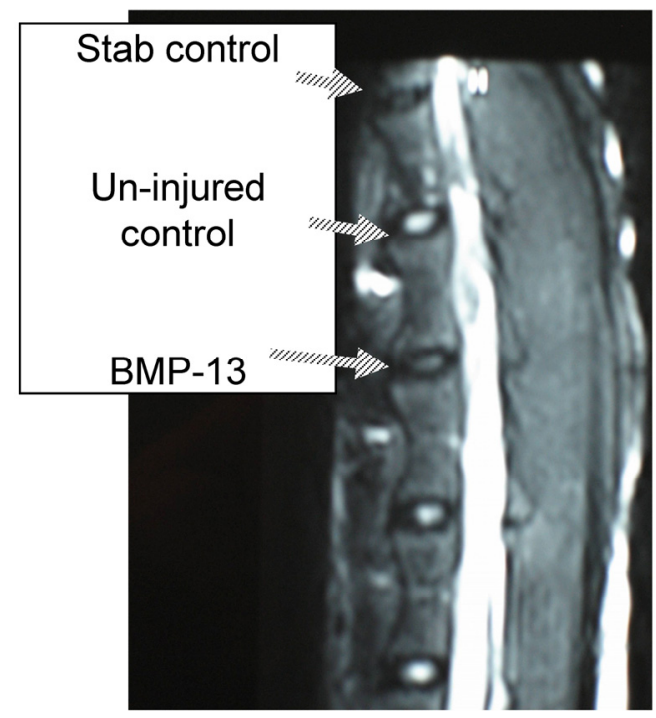

Figure 4. Retention of disc integrity in the presence of BMP13 following annular injury. Magnetic Resonance Image (MRI) scan of sheep spine at 4 months post surgery. Three disc levels are shown: stab control (annular stab with a No. 9 blade followed by injection of 70uL saline solution), Un-injured control (no injury), BMP13 (annular stab with a No. 9 blade followed by injection of 70uL BMP13 solution).

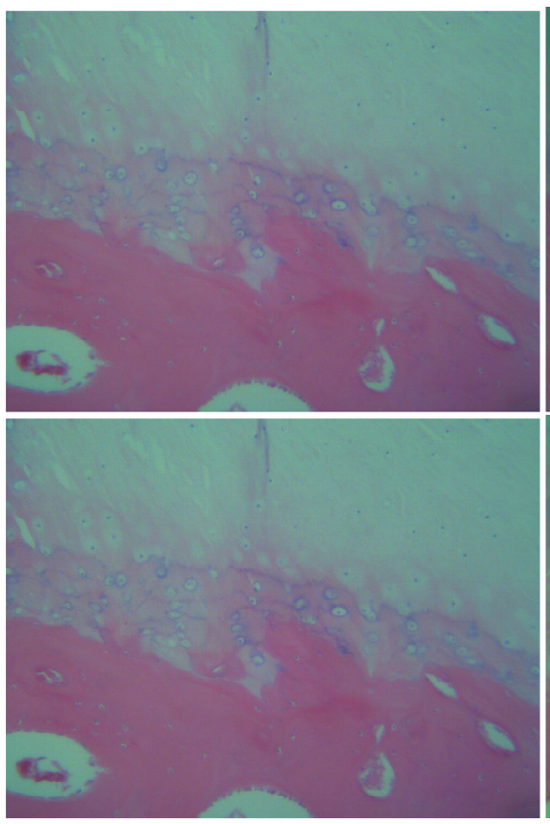

1. Exposed Control - 100x and $400 x$ Images of endplate of sheep $5497+2$ disc

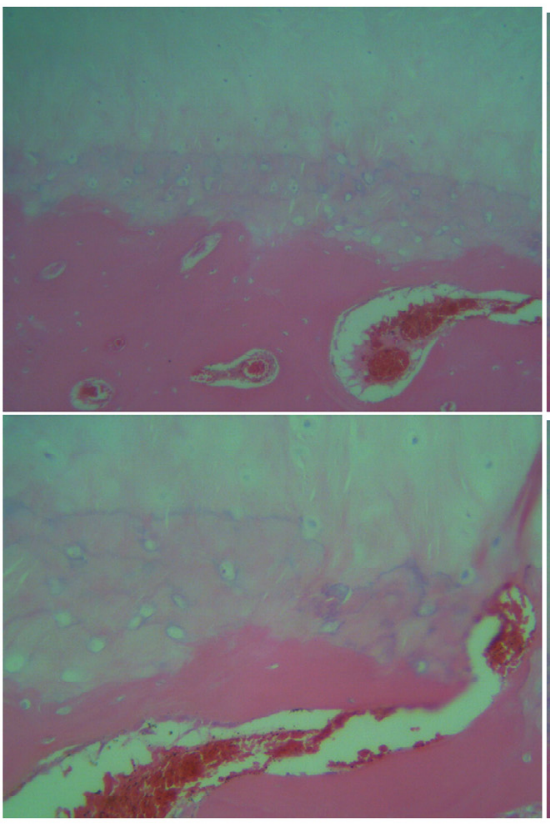

2. Annular stab - saline injected

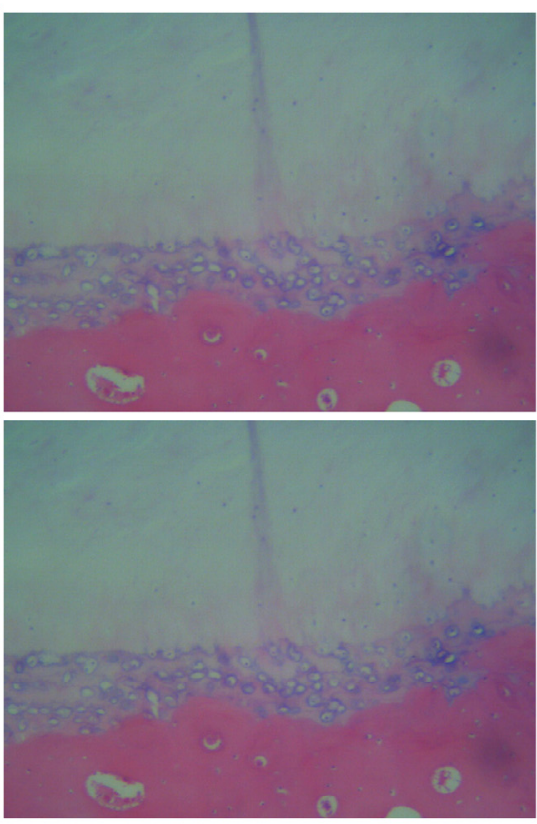

3. Annular stab - BMP13 injection

Figure 5. Increased cellularity of end plate in the presence of BMP13 following annular stab injury. Histological analysis of sheep disc tissues 4 months post surgical injury.

\section{Development}

Members of the BMP family play crucial roles in developmental processes [22,23,65,77-79] and BMP13 is no exception.
BMP13 was simultaneously cloned by Storm et al (1994) by virtue of its homology to other BMP family members using degenerate PCR primers, delineating a BMP sub-family of "Growth and Differentiation Factors" (GDFs 5, 6, 7), so named due to the mutational 
effect of GDF5 gene disruption in causing brachypodism in mice [4]. BMP13 expression was detected in the developing limb - restricted to ossifying long bone centres [3]. The specific role of BMP13 in developmental processes is not yet clear, although its involvement in human eye development has been reported [20]; and homologous genes radar $[18,80]$ and dynamo [81] in Zebrafish; and GDF6 homologues in Xenopus laevis [17] influence early embryonic development.

\section{Embryonic Development}

BMP signalling pathways have been conserved through evolution from Drosophila to mammals, with BMP4 capable of rescuing Drosophila decapentaplegic (dpp) mutations [82]. Similarly early embryonic development, from fruit fly to mammals, is initiated through positional information provided, at least in part, by BMP morphogens and their antagonists forming activity gradients across the embryo $[19,51]$.

Studies of homologous pathways in Zebrafish, Drosophila and Frog (Xenopus laevis) demonstrate a role for BMP13 in early embryo development. In Zebrafish, a BMP13/GDF6 homolog - radar - influences initial dorso-ventral patterning of the embryo as a maternal RNA transcript $[18,83]$. Over-expression of radar in Zebrafish embryos results in a ventralised phenotype with a reduction or absence of dorsal structures [83]. Ablution of maternal transcripts results in a dorsalised Zebrafish embryo phenotype [80], and also results in perturbed ventro-lateral expression of other BMPs, such as BMP2b and BMP4 [80]. A second Zebrafish BMP13/GDF6 homolog, dynamo, is not maternally expressed and has more restricted expression, found in posterior ventral neural tissue, eventually becoming restricted to the ventral spinal cord tissues at the end of somatogenesis, suggesting a more specific role in the organization of the developing spinal cord [81].

It is not known whether the BMP13/GDF6/radar ventralising activity is conserved in other species. Certainly maternal GDF6 (BMP13) RNA transcripts have not been detected in Xenopus embryos [17], although protein translated during oogenesis could conceivably be active in the early frog embryo. However injection of wild type BMP13 (GDF6) into early Xenopus embryos results in a mild ventralisation phenotype [17], similar to that observed in Zebrafish. BMP13/GDF6 induced epidermal genes and inhibited neural markers in Xenopus embryos, activities directly modulated by expression of antagonist molecule, nog$\operatorname{gin}$ [17].

\section{Eye/retinal development}

Studies of human chromosomal abnormalities have recently identified GDF6 (BMP13) within and adjacent to segmental chromosomal deletions in patients with Colobomata [20], a complex series of ocular abnormalities that appear to involve many apparently unrelated genes. Ocular development is also perturbed when zygotic expression of Zebrafish GDF6 homologue radar is inhibited - morphants exhibit reduced eye, head and dorsal neural tube structures [80].

Similarly, BMP13 was also identified as an early regulator of retinal development in a Xenopus model [19]. Phenotypically, depletion of GDF6 resulted in a reduction in eye size, evidence of increased programmed cell death, more disorganized retinal tissue development, and the presence of neural defects in the eye and neural tube $[19,21]$. The influence of BMP13 depletion also extended to wider influences on developing neural tissue and the effects correlated with a decrease in phosphorylated smad $1 / 5 / 8$ signalling molecules, indicative of decreased signalling.

Thus the importance of BMP13/GDF6 and its homologues in embryonic ocular tissue development appears to be conserved across widely variant vertebrate species.

\section{Skeletal development}

As shown in Figure 1 \& 2, BMP13, like all members of the GDF sub-family of BMPs, is highly conserved in vertebrates [10]. All GDF genes are expressed in a stripe pattern in developing joint regions where skeletal segmentation events occur [16,84], with GDF6/7 expression more restricted than GDF5.

GDF6/BMP13 knockout mice are viable and survive to adulthood, however they have abnormal skull joint development and bone fusions at wrist and ankle - sites of major BMP13 developmental expression [84]. Evidence from studying these mice suggested that development of these joints was initiated normally but could not proceed, suggesting a role for BMP13 in the maintenance of developmental processes.

Naturally occurring null mutations in GDF5 cause brachypodism - shortening of long bones, alterations to joint bone formation in wrists, ankles and digits - and are characterized by abnormal cartilage and some bone fusions $[16,85]$. The combination GDF5/6 knockout mouse is far less viable and has additional striking skeletal defects: many limb bones and joints are severely reduced or absent. In addition the vertebral column appears prone to curvature (scoliosis) in many double mutants, with lower thoracic/lumbar vertebrae displaying altered extracellular matrix (proteoglycan) deposition in inter-vertebral chondrocyte-like cells [84]. The authors suggest the GDF knockout mutations exposed a role in joint maintenance and the double knockout may suggest that, rather than representing redundancy of function, 
members of the GDF family co-operate or rely on each other during skeletal development - and potentially in tissue maintenance.

Recently GDF6 was identified as a candidate gene defect present in several familial and sporadic cases of Klippel Feil Syndrome (KFS) [21]. KFS is characterized by heterogeneous congenital defects of the spine, limbs and organ functions $[86,87]$. Breakpoint analysis indicated the involvement of a long range BMP13 regulatory locus in the manifestation of KFS, but also identified two point mutations associated with a familial inherited abnormality (A249E), and two sporadic mutations causing the same syndrome (L289P) (Figure 3) [21]. Both missense mutation sites are located in the pro-domain in the evolutionarily conserved region near the second putative protease cleavage site, further strengthening the possibility that this site is important for the control of BMP13 tissue expression.

\section{BMP13 Antagonists}

BMP antagonists form a protein family that display amino acid homology and evolutionary conservation, possessing a similar "cysteine knot" structure, they are part of the wider TGF- $\beta$ superfamily [15]. In terms of embryo development, early studies with Xenopus and Drosophila found that morphogenic gradients of opposing antagonistic proteins controlled spatial organization in the embryo and led to the establishment of a dorso-ventral axis [88]. BMP antagonists control all aspects of BMP function including effects on tissue re-modelling. They bind to the BMP ligands and prevent interaction with cell surface receptors, thereby blocking intracellular signalling. [25].

The GDF6 homolog in Xenopus was cloned through a gene screen specific for molecules down-regulated by BMP antagonist Noggin, and direct binding of noggin to GDF6 (BMP13) has been demonstrated [17]. Whilst GDF6 was responsible for promoting epidermal tissue modulation, noggin activity induced neural tissues, with normal development the result of a delicate balance between opposing morphogen expression.

Interestingly null noggin mutations have excess cartilage and bone [24]. Excess BMP activity as a result of noggin reduction or antagonism may enhance recruitment of cells to cartilage, resulting in oversized growth plates.

\section{Summary and Future directions}

The highly conserved amino acid sequence of the BMP13 active domain - both within its GDF-subfamily and, quite strikingly, across diverse vertebrate species - suggests a crucial biological function. The literature to date, whilst providing important insights, appears to imply BMP13/GDF6 functions co-operatively and is somewhat redundant, even playing a modulatory role affecting the functions of other BMPs both in development and in adult tissues.

Reports have emerged showing the genetic impact of BMP13 (GDF6) gene disruption in Zebrafish, Xenopus, and in Human individuals; with diverse effects on embryonic ocular and neural tissues, and on the development of skeletal structures such as the skull, limb joints, the developing spinal column and intervertebral discs. Developmental models indicate a very early dorso-ventral patterning function for BMP13/GDF6, which appears to be conserved - at least in fish and amphibians - establishing the early dorso-ventral axis and promoting ventral tissues of the eye, head and neural tube. A conserved role is also apparent in the development of ocular structures. The effect of BMP13 in development seems to lie in its influence upon other members of the BMP family and their antagonists, creating downstream patterns of expression of genes which have documented roles in development of the embryo.

In adult tissues, many studies have shown the stimulatory effects of BMP13 on connective tissue marker gene expression, but BMP13 appears, in most cases, to be no more effective than other GDF's or BMPs, often showing a lesser catabolic effect, at least in vitro. Future studies targeted at understanding the BMP13 signalling pathway would be of interest, particularly using a human protein / tissue combination, to determine specific markers of BMP13 activity aside from other, more extensively characterized BMPs. It would also be of interest to determine the contribution of the second putative protease cleavage site in the BMP13 pro-domain to controlling BMP13 expression and activity - particularly in view of the point mutations that have been identified in this region in conjunction with the manifestation of Klippel-Feil Syndrome and mal-developed intervertebral discs.

There appears to be redundancy in the functions of various GDF and BMP family members such that more than one protein has an effect on a particular tissue. However perhaps these proteins have such precise functions, are expressed in highly specific tissue regions with exact precision, such that what appears to be redundancy is in fact a carefully orchestrated co-operative interplay across the tissue landscape. Morphogenetic gradients created by increasing and decreasing concentrations of morphogens and antagonists that continue to operate in complex tissues, giving them their characteristics and functional delineations. 
Clearly BMP13 function remains an unveiling story, a multi-faceted, pleiotropic morphogen some parts of the vertebrate body just can't do without.

\section{Conflict of Interest}

The authors have declared that no conflict of interest exists.

\section{References}

[1] Urist MR. Bone formation by autoinduction. Science 1965;150:893-9.

[2] Sampath TK, Reddi AH. Dissociative extraction and reconstitution of extracellular matrix components involved in local bone differentiation. Proc Natl Acad Sci U S A 1981;78 (12):7599-603.

[3] Chang SC, Hoang B, Thomas JT, Vukicevic S, Luyten FP, Ryba NJP, Reddi AH, Moos M. Cartilage-derived morphogenic proteins. New Members of the Transforming Growth Factor B superfamily predominantly expressed in long bones during human embryonic development. Journal of Biological Chemistry 1994;269 (45):28227-34.

[4] Storm EE, Huynh TV, Copeland NG, Jenkins NA, Kingsley DM, Lee S-J. Limb alterations in brachypodism mice due to mutations in a new member of the TGF-B superfamily. Nature 1994;368:639.

[5] Raftery LA, Sutherland DJ. TGF-beta family signal transduction in Drosophila development: from Mad to Smads. Dev Biol 1999;210 (2):251-68.

[6] Arora K, Levine MS, O'Connor MB. The screw gene encodes a ubiquitously expressed member of the TGF-beta family required for specification of dorsal cell fates in the Drosophila embryo. Genes Dev 1994;8 (21):2588-601.

[7] Dorfman R, Shilo BZ. Biphasic activation of the BMP pathway patterns the Drosophila embryonic dorsal region. Development 2001;128 (6):965-72.

[8] Ren P, Lim CS, Johnsen R, Albert PS, Pilgrim D, Riddle DL. Control of C. elegans larval development by neuronal expression of a TGF-beta homolog. Science 1996;274 (5291):1389-91.

[9] Suzuki Y, Yandell MD, Roy PJ, Krishna S, Savage-Dunn C, Ross RM, Padgett RW, Wood WB. A BMP homolog acts as a dose-dependent regulator of body size and male tail patterning in Caenorhabditis elegans. Development 1999;126 (2):241-50.

[10] Ducy P, Karsenty G. The family of bone morphogenetic proteins. Kidney Int 2000;57 (6):2207-14.

[11] Reddi HA. Cartilage morphogenesis: role of bone and cartilage morphogenetic proteins, homeobox genes and extracellular matrix. Matrix Biology 1994;14 (8):599-606.

[12] Wozney JM. The bone morphogenic protein family and osteogenesis. Molecular Reproduction and Development 1992;32:160-7.

[13] Wozney JM, Rosen V. Bone morphogenetic protein and bone morphogenetic protein gene family in bone formation and repair. Clin Orthop Relat Res 1998 (346):26-37.

[14] Reddi AH. Interplay between bone morphogenetic proteins and cognate binding proteins in bone and cartilage development: noggin, chordin and DAN. Arthritis Res 2001;3 (1):1-5.

[15] Yanagita M. BMP antagonists: their roles in development and involvement in pathophysiology. Cytokine Growth Factor Rev 2005;16 (3):309-17.

[16]Storm EE, Kingsley DM. Joint patterning defects caused by single and double mutations in members of the bone morphogenetic protein (BMP) family. Development 1996;122 (12):3969-79.

[17] Chang C, Hemmati-Brivanlou A. Xenopus GDF6, a new antagonist of noggin and a partner of BMPs. Development 1999;126 (15):3347-57.
[18]Rissi M, Wittbrodt J, Delot E, Naegeli M, Rosa FM. Zebrafish Radar: a new member of the TGF-beta superfamily defines dorsal regions of the neural plate and the embryonic retina. Mech Dev 1995;49 (3):223-34.

[19] Hanel ML, Hensey C. Eye and neural defects associated with loss of GDF6. BMC Developmental Biology 2006;6:43-56.

[20] Asai-Cloakwell M, French CR, Berry KM, Ye M, Koss R, Somerville M, Mueller R, Heyningen Vv, Waskiewicz AJ, Lehmann OJ. GDF6, a novel locus for a spectrum of ocular developmental anomalies. The Americal Journal of Human Genetics 2007;80:306.

[21]Tassabehji M, Fang ZM, Hilton EN, McGaughran J, Zhao Z, de Bock CE, Howard E, Malass M, Donnai D, Diwan A, Manson FD, Murrell D, Clarke RA. Mutations in GDF6 are associated with vertebral segmentation defects in Klippel-Feil syndrome. Human Mutation 2008;29 (8):1017-27.

[22] Wan M, Cao X. BMP signaling in skeletal development. Biochemical and Biophysical Research communications 2005;328:651-7.

[23] Canalis E, Economides AN, Gazzerro E. Bone morphogenetic proteins, their antagonists, and the skeleton. Endocr Rev 2003;24 (2):218-35.

[24] Balemans W, Van Hul W. Extracellular regulation of BMP signaling in vertebrates: a cocktail of modulators. Dev Biol 2002;250 (2):231-50.

[25] Abe E. Function of BMPs and BMP antagonists in adult bone. Ann NY Acad Sci 2006;1068:41-53.

[26] Cui Y, Jean F, Thomas G, Christian JL. BMP4 is proteolytically activated by furin and/or PC6 during vertebrate embryonic development. The EMBO Journal 1998;17 (16):4735-43.

[27] Degnin C, Jean F, Thomas G, Christian JL. Cleavages within the prodomain direct intracellular trafficking and degradation of bone morphogenetic protein-4. Molecular Biology of the Cell 2004;15 (11):5012-20.

[28] Sopory S, Nelsen SM, Degnin C, Wong C, Christian JL. Regulation of bone morphogenetic protein- 4 activity by sequence elements within the prodomain. Journal of Biological chemistry 2006;281 (45):34021-31.

[29] Cui Y, Hackenmiller R, Berg L, Jean F, Nakayama T, Thomas G, Christian JL. The activity and signalling range of mature BMP-4 is regulated by sequential cleavage at two sites within the prodomain of the precursor. Genes \& Development 2001;15 (21):2797-802.

[30]Gregory KE, Ono RN, Charbonneau NL, Kuo CL, Keene DR, Bachinger HP, Sakai LY. The prodomain of BMP7 targets the BMP7 complex to the extracellular matrix. Journal of Biological chemistry 2005;280 (30):27970-80.

[31] McDonald NQ, Hendrickson WA. A structural superfamily of growth factors containing a cystine knot motif. Cell 1993;73 (3):421-4.

[32] Constam DB, Calfon M, Robertson EJ. SPC4, SPC6 and the novel protease SPC7 are coexpressed with bone morphogenic proteins at distinct sites during embryogenesis. Journal of Cellular Biology 1996;134 (1):181-91.

[33]Rockwell NC, Krysan DJ, Komiyama T, Fuller RS. Precursor processing by Kex2/Furin proteases. Chemical Reviews 2002;102:4525-48.

[34] Erlacher L, McCartney J, Piek E, Dijke PT, Yanagishita M, Opermann H, Luyten FP. Cartilage derived morphogenic proteins and osteogenic protein-1 differentially regulate osteogenesis. Journal of Bone and Mineral Research 1998;13 (3):383-92.

[35] Erlacher L, Ng C, Ullrich R, Krieger S, Luyten FP. Presence of cartilage-derived morphogenic proteins in articular cartiolage and enhancement of matrix replacement in vitro. Arthritis \& Rheumatism 1998;41 (2):263-73.

[36] Bobacz K, Gruber R, Soleiman A, Graninger WB, Luyten FP, Erlacher L. Cartilage derived morphogenic protein-1 and -2 are 
endogenously expressed in healthy and osteoarthritic human articular chondrocytes and stimulate matrix synthesis. Osteoarthritis \& Cartilage 2002;10:394-401.

[37] Bobacz K, Ullrich R, Amoyo L, Erlacher L, Smolen JS, Graninger WB. Stimulatory effects of distinct members of the bone morphogenic protein family on ligament fibroblasts. Ann Rheum Dis 2006;65:169-77.

[38] Wozney JM, Rosen V, Celeste AJ, Mitsock LM, Whitters MJ, Kriz RW, Hewick RM, Wang EA. Novel regulators of bone formation: molecular clones and activities. Science 1988;242 (4885):1528-34.

[39]Sampath TK, Coughlin JE, Whetstone RM, Banach D, Corbett C, Ridge RJ, Ozkaynak E, Oppermann H, Rueger DC. Bovine osteogenic protein is composed of dimers of OP-1 and BMP-2A, two members of the transforming growth factor-beta superfamily. J Biol Chem 1990;265 (22):13198-205.

[40] Aono A, Hazama M, Notoya K, Taketomi S, Yamasaki H, Tsukuda R, Sasaki S, Fujisawa Y. Potent ectopic bone-inducing activity of bone morphogenetic protein- $4 / 7$ heterodimer. Biochem Biophys Res Commun 1995;210 (3):670-7.

[41] Hazama M, Aono A, Ueno N, Fujisawa Y. Efficient expression of a heterodimer of bone morphogenetic protein subunits using a baculovirus expression system. Biochem Biophys Res Commun 1995;209 (3):859-66.

[42] Suzuki A, Kaneko E, Maeda J, Ueno N. Mesoderm induction by BMP-4 and -7 heterodimers. Biochem Biophys Res Commun 1997;232 (1):153-6.

[43] Nishimatsu S, Thomsen GH. Ventral mesoderm induction and patterning by bone morphogenetic protein heterodimers in Xenopus embryos. Mech Dev 1998;74 (1-2):75-88.

[44] Nohe A, Keating E, Knaus P, Petersen NO. Signal transduction of bone morphogenetic protein receptors. Cellular Signalling 2004;16 (3):291-9.

[45] Yamashita H, Ten Dijke P, Heldin CH, Miyazono K. Bone morphogenetic protein receptors. Bone 1996;19 (6):569-74.

[46] Miyazono K. Signal transduction by bone morphogenetic protein receptors: functional roles of Smad proteins. Bone 1999;25 (1):91-3.

[47] Carcamo J, Weis FM, Ventura F, Wieser R, Wrana JL, Attisano L, Massague J. Type I receptors specify growth-inhibitory and transcriptional responses to transforming growth factor beta and activin. Mol Cell Biol 1994;14 (6):3810-21.

[48] Kawakami $Y$, Ishikawa $T$, Shimabara $M$, Tanda N, Enomoto-Iwamoto M, Iwamoto M, Kuwana T, Ueki A, Noji S, Nohno T. BMP signaling during bone pattern determination in the developing limb. Development 1996;122 (11):3557-66.

[49] von Bubnoff A, Cho KW. Intracellular BMP signaling regulation in vertebrates: pathway or network? Dev Biol 2001;239 (1):1-14.

[50]Sakou T. Bone morphogenetic proteins: from basic studies to clinical approaches. Bone 1998;22 (6):591-603.

[51] Wilm TP, Solnica-Krezel L. Radar breaks the fog: insights into dorsoventral patterning in zebrafish. Proc Natl Acad Sci U S A 2003;100 (8):4363-5.

[52] Massague J. How cells read TGF-beta signals. Nat Rev Mol Cell Biol 2000;1 (3):169-78.

[53] Mazerbourg S, Sangkuhl K, Juo CW, Sudo S, Klein C, Hsueh AJW. Identification of receptors and signalling pathways for orphan bone morphogenic protein/growth differentiation factor ligands based on genomic analyses. Journal of Biological Chemistry 2005;280 (37):32122-32.

[54]Zoricic S, Maric I, Bobinac D, Vukicevic S. Expression of bone morphogenic proteins and cartilage-derived morphogenic proteins during osteophyte formation in humans. . Journal of Anatomy 2003;202:269-77.

[55] Tomaski SM, Zalzal GH. In vitro regulation of expression of cartilage derived morphogenetic proteins by growth hormone and insulin-like growth factor 1 in the bovine cricoid chondrocyte. Arch Otolaryngol Head Neck Surg 1999;125:901-6.
[56]Gooch KJ, Bunk T, Courter DL, Sieminski AL, Vunjak-Novakovic G, Freed LE. Bone morphogenic proteins -2, -12, -13 modulate in vitro development of engineered cartilage. Tissue Engineering 2002;8 (4):591-601.

[57] Gruber R, Mayer C, Bobacz K, Graninger W, Luyten FP, Erlacher L. Effects of cartilage-derived morphogenetic proteins and osteogenic protein-1 on osteochondrogenic differentiation of periosteum-derived cells. Endocrinology 2001;142 (5):2087-94.

[58] Nochi H, Sung JH, Lou J, Adkisson HD, Maloney WJ, Hruska KA. Adenovirus mediated BMP-13 gene transfer induces chondrogenic differentiation of murine mesenchymal progenitor cells. Journal of Bone and Mineral Research 2004;19 (1):111-22.

[59] Yeh L-CC, Tsai AD, Zavala MC, Lee JC. Cartilage-derived proteins enhance the osteogenic protein-1-induced osteoblastic cell differentiation of $\mathrm{C} 2 \mathrm{C} 12$ cells. Journal of Cellular Physiology 2004;201:401-8.

[60] Wolfman NM, Hattersley G, Cox K, Celeste AJ, Nelson R, Yamaji N, Dubem JL, DiBlasio-Smith E, Nove J, Song JJ, Wozney JM, Rosen V. Ectopic induction of tendon and ligament in rats by growth and differentiation factors 5, 6 and 7, members of the TGFB gene family. Journal of Clinical Investigation 1997;100:321-3.

[61] Forslund C, Rueger D, Aspenberg P. A comparative dose-response study of cartilage-derived morphogenic protein (CDMP) -1, -2, -3 for tendon healing in rats. Journal of Orthopedic Research 2003;21:617-21.

[62] Gruber R, Mayer C, Schulz W, Graninger W, Peterlik M, Watzek G, Luyten FP, Erlacher L. Stimulatory effects of cartilage derived morphogenetic proteins -1 and -2 on osteogenic differentiation of bone marrow stromal cells. Cytokine 2000;12 (11):1630-8.

[63] Yeh L-CC, Tsai AD, Lee JC. Cartilage-derived morphogenic proteins induce osteogenic gene expression in the $\mathrm{C} 2 \mathrm{C} 12$ mesenchymal cell line. Journal of Cellular Biochemistry 2005;95:173-88.

[64] Li J, Kim KS, Park JS, Elmer WA, Hutton WC, Yoon ST. BMP-2 and CDMP-2: stimulation of chondrocyte production of proteoglycan. Journal of Orthopedic Science 2003;8:829-35.

[65] Reddi AH. Bone morphogenetic proteins and skeletal development: the kidney-bone connection. Pediatr Nephrol 2000;14 (7):598-601.

[66]Xiao YT, Xiang LX, Shao JZ. Bone morphogenetic protein. Biochem Biophys Res Commun 2007;362 (3):550-3.

[67] Chuen FS, Chuk CY, Ping WY, Nar WW, Kim HL, Ming CK. Immunohistochemical characterization of cells in adult human patellar tendons. J Histochem Cytochem 2004;52 (9):1151-7.

[68] Wong YP, Fu SC, Cheuk YC, Lee KM, Wong MWN, Chan KM. Bone morphogenetic protein 13 stimulates cell proliferation and production of collagen in human patellar tendon fibroblasts. Acta Orthopaedica 2005;76 (3):421-7.

[69] Forslund C, Aspenberg P. CDMP-2 indices bone or tendon-like tissue depending on mecanical stimulation. Journal of Orthopedic Research 2002;20:1170-4.

[70] Forslund C, Aspenberg P. Tendon healing stimulated by injected CDMP-2. Med Sci Sports Exerc 2001;33 (5):685-7.

[71]Helm GA, Li JZ, Alden TD, Hudson SB, Beres EJ, Cunningham M, Mikkelsen MM, Pittman DD, Kerns KM, Kallmes DF. A light and electron microscopic study of ectopic tendon and ligament formation induced by bone morphogenetic protein-13 adenoviral gene therapy. J Neurosurg 2001;95 (2):298-307.

[72]Zhang Y, An HS, Thonar EJ-MA, Chubinskaya S, He T-C, Phillips FM. Comparative effects of bone morphogenetic proteins and SOX9 overexpression on the extracellular matrix metabolism of bovine nucleus pulposus cells. Spine 2006;31 (19):2173-9.

[73]Zhang Y, Anderson G, Phillips FM, Thonar EJ-M, He T-C, Pietryla D, An HS. Comparative effects of bone morphogenetic proteins and SOX9 overexpression on matrix accumulation by 
bovine annulus fibrosus cells. Implications for annular repair. Spine 2007;32 (23):2515-20.

[74]Zhang Y, Li Z, Thonar EJ-MA, An HS, He T-C, Pietryla D, Phillips FM. Transduced bovine articular chondrocytes affect the metabolism of co-cultured nucleus pulposus cells in vitro: implications for chondrocyte transplantation into the intervertebral disc. Spine 2005;30 (23):2601-7.

[75] Osti OL, Vernon-Roberts B, Fraser RD. Annulus tears and intervertebral disc degeneration. An experimental study using an animal model. Spine 1990;15 (8):762-7.

[76]Moore RJ, Osti OL, Vernon-Roberts B, Fraser RD. Changes in endplate vasculalrity after an outer annulus tear in the sheep. Spine 1992;17 (8):874-7.

[77] Wordinger RJ, Clark AF. Bone morphogenetic proteins and their receptors in the eye. Exp Biol Med (Maywood) 2007;232 (8):979-92.

[78] Dale L, Wardle FC. A gradient of BMP activity specifies dorsal-ventral fates in early Xenopus embryos. Semin Cell Dev Biol 1999;10 (3):319-26.

[79] Wang YC, Ferguson EL. Spatial bistability of Dpp-receptor interactions during Drosophila dorsal-ventral patterning. Nature 2005;434 (7030):229-34.

[80]Sidi S, Goutel C, Peyrieras N, Rosa FM. Maternal induction of ventral fate by zebrafish radar. Proc Natl Acad Sci U S A 2003;100 (6):3315-20.

[81] Bruneau S, Rosa FM. Dynamo, a new zebrafish DVR member of the TGF-beta superfamily is expressed in the posterior neural tube and is up-regulated by Sonic hedgehog. Mech Dev 1997;61 (1-2):199-212.

[82] Padgett RW, Wozney JM, Gelbart WM. Human BMP sequences can confer normal dorsal-ventral patterning in the Drosophila embryo. Proc Natl Acad Sci U S A 1993;90 (7):2905-9.

[83] Goutel C, Kishimoto Y, Schulte-Merker S, Rosa F. The ventralizing activity of Radar, a maternally expressed bone morphogenetic protein, reveals complex bone morphogenetic protein interactions controlling dorso-ventral patterning in zebrafish. Mech Dev 2000;99 (1-2):15-27.

[84] Settle SH, Rountree RB, Sinha A, Thacker A, Higgins K, Kingsley DM. Multiple joint and skeletal patterning defects caused by single and double mutations in the mouse gdf6 and gdf5 genes. . Developmental Biology 2003;254:116-30.

[85]Storm EE, Kingsley DM. GDF5 coordinates bone and joint formation during digit development. Dev Biol 1999;209 (1):11-27.

[86]Clarke RA, Catalan G, Diwan A, Kearsley JH. Heterogeneity in Klippel-Feil Syndrome: a new classification. Paediatric Radiology 1998;28:967-74.

[87] Clarke RA, Kearsley JH, Walsh DA. Patterned expression in familial Klippel-Feil Syndrome. Teratology 1996;53:152-7.

[88] Barth KA, Kishimoto Y, Rohr KB, Seydler C, Schulte-Merker S, Wilson SW. Bmp activity establishes a gradient of positional information throughout the entire neural plate. Development 1999;126 (22):4977-87. 